\title{
Roles of exogenously regulated bFGF expression in angiogenesis and bone regeneration in rat calvarial defects
}

\author{
MEILING CHEN, KE SONG, NIANJING RAO, MENGQI HUANG, ZHENGJIANG HUANG and YINGGUANG CAO
}

\author{
Center of Stomatology, Tongji Hospital, Tongji Medical College, Huazhong \\ University of Science and Technology, Wuhan 430030, P.R. China
}

Received November 15, 2010; Accepted January 13, 2011

DOI: 10.3892/ijmm.2011.619

\begin{abstract}
Regulation of transgene expression and function is important for gene therapy because it allows complex biological processes to be controlled and monitored. Basic fibroblast growth factor (bFGF) is an effective angiogenic factor and bone regeneration factor; it can induce differentiation of mesenchymal stem cells (MSCs) in vitro and bone regeneration in vivo. Further, exogenous regulation of controllable bFGF expression in the bone regeneration area safely allows bone formation and regeneration. In our study, we constructed a recombinant adeno-associated virus type 2 (rAAV2)-based bFGF gene delivery system, which is regulated by tetracycline or doxycycline (Dox, an analogue of tetracycline). We evaluated the regulatory effects of this system on bFGF transgenic expression in vitro and in vivo. We found that bFGF could increase the mRNA expression levels of osteoblast differentiation factor and the activity of alkaline phosphatase (ALP). Dox could effectively regulate bFGF expression, thus controlling MSC differentiation. After in vivo transplantation of genetically engineered MSCs, animals not treated with Dox showed significant bone formation and angiogenesis compared with the group treated with Dox. Dox may also effectively regulate angiogenesis and bone regeneration in vivo. Therefore, the inducible bFGF system is an effective way of regulating bone regeneration and formation.
\end{abstract}

\section{Introduction}

Autogenous bone, allograft bone, guided bone regeneration, and other traditional methods for treatment of craniofacial defects have achieved acceptable outcomes $(1,2)$. However, the newly generated bone is not comparable to the original bone. Consequently, gene-enhanced tissue engineering methods are gaining increased attention (3-5).

Correspondence to: Dr Yingguang Cao, Center of Stomatology, Tongji Hospital, Tongji Medical College, Huazhong University of Science and Technology, Wuhan 430030, P.R. China

E-mail: cyg0729@tjh.tjmu.edu.cn

Key words: basic fibroblast growth factor, gene regulation system, gene therapy, angiogenesis, bone regeneration
Bone metabolism is mainly mediated through local or systemic factors (6). Of these factors, basic fibroblast growth factor (bFGF) is one of the most effective osteogenic and angiogenic growth factors involved in recruiting mesenchymal and osteogenic cells and stimulating their proliferation and differentiation (7). More importantly, bFGF can promote angiogenesis, which accelerates bone healing (7-9). Angiogenesis not only provides nutrition via metabolic pathways but is also an important process of obtaining repair factors, which play an important role in intramembranous ossification (10). If applied directly, large doses of bFGF protein are required. In addition, the bFGF protein has a short half-life, and repeated administration has achieved limited clinical success. Therefore, long-term stable expression of the transferred gene is a more attractive alternative.

Viral vectors have been proven to be effective for gene therapy. rAAV2 can infect both mitotic and post-mitotic cells of various types, producing stable gene expression without inducing toxic and immune responses (11). Considering these advantages and its tissue-specific features, rAAV2-mediated gene transfer is widely used for bone and cartilage studies $(11,12)$. Transgenic regulation is particularly important in gene therapy. Time and dose regulation of exogenous gene expression can circumvent abnormalities resulting from sustained expression after tissue repair. Therefore, it is necessary to regulate transgene expression, in particular, to suppress transgene expression. The tetracycline (Tet) regulatory system $(11,13,14)$, which has high efficiency and low toxicity, contains two components: the transactivator, tTA, and the Tet-regulated element (TRE). In the Tet-off system (14), tTA binds TRE and activates transgene expression in the absence of tet or the tet analog, doxyccyline (Dox). In the presence of Dox, however, tTA separates from TRE, and transgene expression is shut off.

MSCs have multiple differentiation potentials and can differentiate into osteoblasts under specific environmental conditions, making them the most ideal seed cells for bone tissue engineering (15). Osteoblast precursor cells can not only increase the number of osteoblasts, a requirement for bone regeneration, but also mediate the production of transgenic paracrine growth factors that stimulate the proliferation and differentiation of host cells. Bone graft materials, such as $\beta$-tricalcium phosphate ( $\beta$-TCP), have been widely used to repair bone defects in clinical and experimental settings (16). 
$\beta$-TCP mimics the extracellular matrix and has bone conduction features. When implanted in the body along with transgenic cells for the purpose of bone induction, the transgenic cells can be regulated and protected, and bone regeneration can be promoted through transgene expression. Considering the important roles that bFGF plays in angiogenesis and osteogenic differentiation, we used genetically enhanced bone tissue engineering to investigate if regulating bFGF expression can control angiogenesis and bone regeneration.

\section{Materials and methods}

Production and purification of rAAV-tet-off-bFGF. The pBluescript SKII-bFGF plasmid, which contains the complete coding sequence (CDS) of mouse bFGF (465 bp), was provided by RIKEN BioResource Center (Japan). Plasmids pAAV-S3KzkhrGFP and pSVneo were provided by the Vector Gene Technology Company limited (VGTC, China). The vector pAAV-S3-KzkhrGFP has characteristics of both the AAV and Tet-off systems. In the pAAV-S3-KzkhrGFP plasmid, the expression of the green fluorescence protein (GFP) is controlled by Dox. In the presence of Dox, GFP expression is shut down, while in the absence of Dox, GFP expression is turned on. We replaced GFP with the coding sequence of bFGF so that bFGF expression was controlled by Dox. A 480 bp-fragment coding the full-length CDS of bFGF was cut from pBluescript SKII-bFGF by EcoRV and NotI (Takara, Tokyo, Japan) double digestion; this fragment was then ligated to pAAV-S3-KzkhrGFP, giving rise to the recombinant $\mathrm{pAAV}-\mathrm{S} 3-\mathrm{bFGF}$ plasmid.

pAAV-S3-bFGF and pSVneo were co-transfected into BHK-21 cells using Lipofectamine 2000 (Invitrogen, Carlsbad, CA, USA) according to the manufacturer's instructions. G418resistant BHK-21 clones were transfected with HSV1-rc/DUL2. The infected cells were processed using chloroform treatment, PEG 8000/ $\mathrm{NaCl}$ precipitation, and chloroform extraction for purification. The digoxin-labeled CBA probe dot blot method (17) was used to measure the physical titer of rAAV2tet-off-bFGF, and the result was $1.8 \times 10^{12}$ genomes $/ \mathrm{ml}$. All preparations were free of infectious adenovirus and wild-type AAV contamination.

Isolation and culture of MSCs. The femoral and tibial bone marrow cavity of 3-month male SD rats (The Animal Center, Tongji Hospital, Huazhong University of Science and Technology, China) was rinsed with low-glucose DMEM (Gibco, Grand Island, NY, USA) supplemented with 10\% fetal bovine serum (FBS) (Gibco), $2 \mathrm{mM}$ glutamine (Gibco), penicillin/streptomycin $(100 \mathrm{U} / \mathrm{ml}$ and $100 \mathrm{mg} / \mathrm{ml}$, respectively) to obtain MSCs. The MSCs were cultured at $37^{\circ} \mathrm{C}$ in $5 \% \mathrm{CO}_{2}$, and the medium was renewed every 3 days. The cells were washed with PBS twice and treated with $0.25 \%$ trypsinEDTA (Gibco) for digestion and during passaging. The third generation of cells was used in our study.

In vitro infection and Dox treatment of MSCs. A 6-well plate was seeded with $1 \times 10^{6}$ MSCs cells per well. After $24 \mathrm{~h}$ of culture, the MSCs were transduced with rAAV2-tet-off-bFGF $\left(\mathrm{MOI}=1 \times 10^{5}\right)$. To determine the appropriate dose of Dox for controlling bFGF expression, the MSCs were treated with 0, 0.5, 1, or $2 \mu \mathrm{g} / \mathrm{ml}$ Dox (Cat. No. D9891, Sigma, St. Louis,
Table I. Primer pairs used for the real-time RT-PCR.

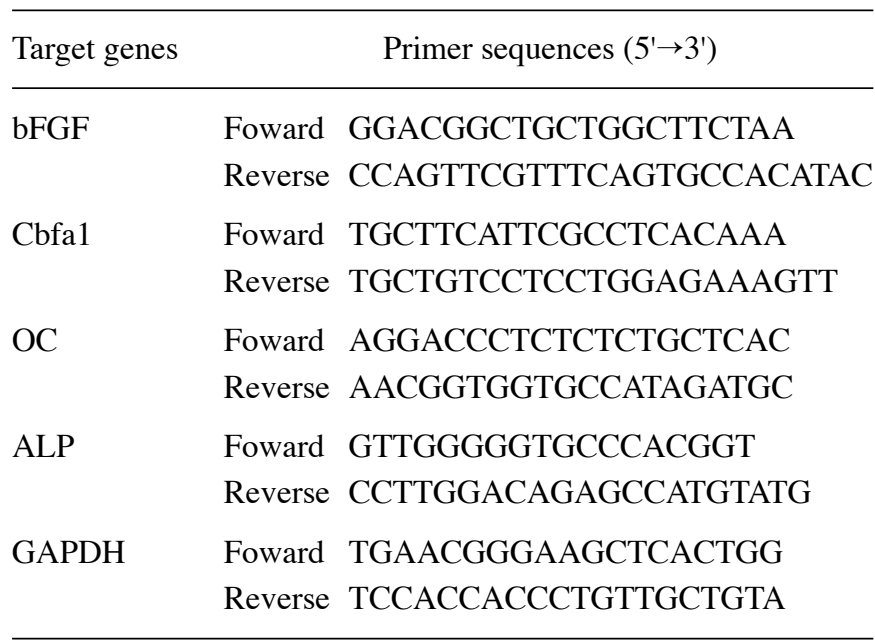

MO, USA). The MSCs that were not transduced were used as controls. The medium was renewed every 1 day in the presence or absence of Dox. After 7 days of Dox treatment, total RNA and total protein were extracted.

Real-time RT-PCR analysis. Total RNA was isolated from cultured MSCs 7 days after Dox treatment (Dox treatment was initiated $24 \mathrm{~h}$ post-transduction) with an RNeasy kit (Invitrogen, Carlsbad, CA, USA). The first-strand of the cDNA was generated using SuperScript II (Invitrogen). Real-time RT-PCR was performed in a LightCycler-FastStart DNA Master SYBR-Green system (Roche, Tokyo, Japan). The primer pairs of bFGF are listed in Table I.

Western blotting. The regulation of bFGF protein levels by Dox was assessed by Western blotting. All cell samples were harvested by applying the loading buffer directly over the cell monolayers after 3 washes with PBS. Electrophoresis and transfer of proteins onto nitrocellulose membranes and blocking of membranes were performed using standard procedures. Rabbit polyclonal antibodies against bFGF (R\&D Systems, Minneapolis, MN, USA) (1:500) were incubated with the membranes overnight at $4^{\circ} \mathrm{C}$. A rat anti-rabbit IgG secondary antibody conjugated to horseradish peroxidase (R\&D Systems) was used at a dilution of 1:1000 and incubated with membranes for $1 \mathrm{~h}$ at room temperature. Proteins were visualized by ECL chemiluminescence (Amersham, Piscataway, NJ, USA).

Alkaline phosphatase (ALP) assay and real-time RT-PCR analysis. The differentiation of transduced MSCs under basal conditions (+Dox) and induced conditions (-Dox) were evaluated using an ALP kit (Sigma) $24 \mathrm{~h}$ and 14 days after Dox treatment, respectively. Next, $1 \mu \mathrm{g} / \mathrm{ml}$ of Dox obtained from the results of the frontal experiment was used to inhibit the expression of bFGF. ALP activity was standardized to the protein content, and the results were reported as $\mathrm{mM} \mathrm{pNP} / \mathrm{mg}$ protein. In addition, the expressions of osteocalcin (OC), core binding factor 1 (Cbfa1), and ALP were detected by real-time RT-PCR after 7 days of treatment with Dox. The target genes and their primers are shown in Table I. 
Preparation of MSCs $/ \beta$-TCP complexes. $\beta$-TCP graft materials $\left(\beta\right.$-TCP, Cerasorb ${ }^{\circledast}$, pore diameter $\left.500-1000 \mu \mathrm{m}\right)$ were provided by Curasan Company (Germany). The granules were pre-wettted with culture medium in 6-well plates. The treated group was rAAV2-tet-off-bFGF-transduced MSCs with Dox, and the untransduced MSCs were used as controls. Next, we added $100 \mathrm{mg}$ of $\beta$-TCP per well diluted in DMEM to the 6-well plates for pre-inoculation, and $1 \times 10^{6}$ cells were carefully seeded $24 \mathrm{~h}$ later. The cell-scaffold complexes were cultured for $2 \mathrm{~h}$ at $37^{\circ} \mathrm{C}$ in $5 \% \mathrm{CO}_{2} / 95 \%$ air for better cell attachment. Then, $2 \mathrm{ml}$ of DMEM medium was slowly added around the complexes. After 15 or $72 \mathrm{~h}$ later, the composites were fixed in $4 \%$ paraformaldehyde and prepared for scanning electron microscopy (SEM) (Hitachi, Tokyo, Japan) observation. After 24 h, 7 days or 14 days of composition, the cell activity was measured using the MTS CellTiter 96 AQueous non-radioactive cell proliferation assay (Promega, Madison, WI, USA). The adherent MSCs that were not bound to $\beta$-TCP were maintained as the negative controls. The mRNA levels of osteogenic genes (OC, Cbfa1, and ALP) were detected by real-time RT-PCR after three-dimensional culture. The sequences of the primers and the condition of the reactions were identical to those of the frontal experiments.

In vivo experimental model. Eighty 12 -week-old male SD rats were anesthetized with a combination of ketamine $(40 \mathrm{mg} / \mathrm{kg})$ and xylazine $(5 \mathrm{mg} / \mathrm{kg})$. Next, a calvarial arc incision was made, cranial skin flaps and muscular layers were elevated, and the mucoperiosteum was completely reflected. A saline-cooled trephine drill (NSK, Tokyo, Japan) was used to create a critical-sized ( $8 \mathrm{~mm}$, diameter) circular and transosseous defect on the cranium. Animals were randomly divided into 4 groups: normal (group A); $\beta$-TCP (group B); MSCs $/ \beta$-TCP composite group (group C); and rAAV2-tetoff-bFGF-transduced MSCs and $\beta$-TCP composite group (group D). Each group was further divided into Dox treated (+Dox) and non-Dox groups (-Dox). Defects were filled with $100 \mathrm{mg} \beta$-TCP composite with $1 \times 10^{6}$ cells. The composite was carefully covered with mucoperiosteum to avoid displacement. The flap was repositioned and sutured. The animals' drinking water was supplemented with Dox $(0.5 \mathrm{mg} / \mathrm{ml}$ in $5 \%$ sucrose, changed every $48 \mathrm{~h}$ ) to activate the tet-off system in order to inhibit bFGF expression. The animals were euthanized 2 and 8 weeks after surgery, for micro-CT and histological examination. Animal studies were approved by our University Ethics Committee.

Micro-CT. Bone formation was measured using a Micro-CT ( $\mu \mathrm{CT} 40$, Scanco, Switzerland). Images were reconstructed at a resolution of $20 \mu \mathrm{m}$ in order to visualize the volume of new bone formation and quantitatively determine the bone volume fraction (Bone Volume/Total Volume) of the newly formed bone.

Polychrome sequential labeling. Polysequential labeling was used to observe bone deposition. At 4, 8, 12 and 14 days after transplantation in the 2 weeks the -Dox group D, a sequence of fluorochrome labels with calcein green $(10 \mathrm{mg} / \mathrm{kg}$ body weight), doxycycline ( $30 \mathrm{mg} / \mathrm{kg}$ body weight), alizarin complexone $(30 \mathrm{mg} / \mathrm{kg}$ body weight), and xylenol-orange (90 mg/kg body weight) were administered by intravenous

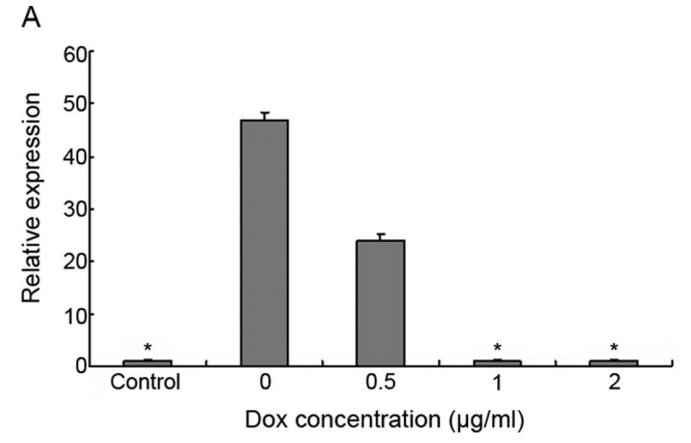

B

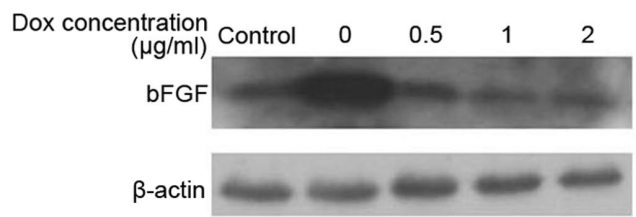

C

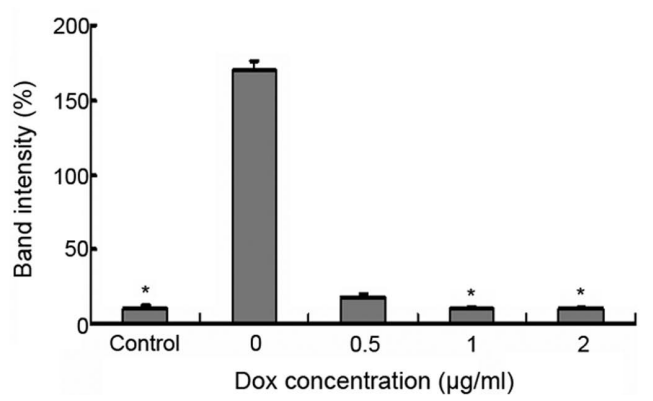

Figure 1. The different concentrations of Dox affecting the control of bFGF expression. (A) After 7 days of Dox treatment, the expression level of bFGF mRNA was detected by real-time RT-PCR. (B) Western blot analysis was used to detect bFGF protein expression in transduced MSCs with Dox regulation. (C) The band intensity was determined by semi-quantitative analysis. MSCs that were not transduced served as controls. rAAV2-tet-offbFGF-transduced MSCs treated with $0,0.5,1$, and $2 \mu \mathrm{g} / \mathrm{ml}$ Dox. ${ }^{*} \mathrm{P}<0.01$ indicates significant differences compared with the group of rAAV2-tet-offbFGF-transduced MSCs without Dox treatment.

injection. In the 8-week groups, the following fluorochromes were intraperitoneally injected at $14,11,7$, and 3 days before tissue sampling: xylenolorange, calcein green, doxycycline, and alizarin complexone. All the fluorochromes were purchased from Sigma.

Histological examination. After conventional fixation, the specimens were embedded in paraffin, and 5- $\mu \mathrm{m}$ sections were obtained. Hematoxylin and eosin (H\&E) and Masson's trichrome staining were performed. For the immunohistochemical study of FactorVIII-related antigen, a marker of vascular endothelial cells, the sections were incubated in rabbit anti-FactorVIII-related antigen (R\&D Systems), followed by incubation in horseradish peroxidase-conjugated goat antirabbit IgG (R\&D Systems). The staining was observed with a BX51 camera (Olympus, Tokyo, Japan). The staining intensities of the new bone and angiogenesis regions were quantified with Image-Pro Plus 6.0 software (Media Cybernetics).

Statistical analysis. All the experiments were repeated at least 3 times, and the data are expressed as mean \pm SEM. SPSS 
A
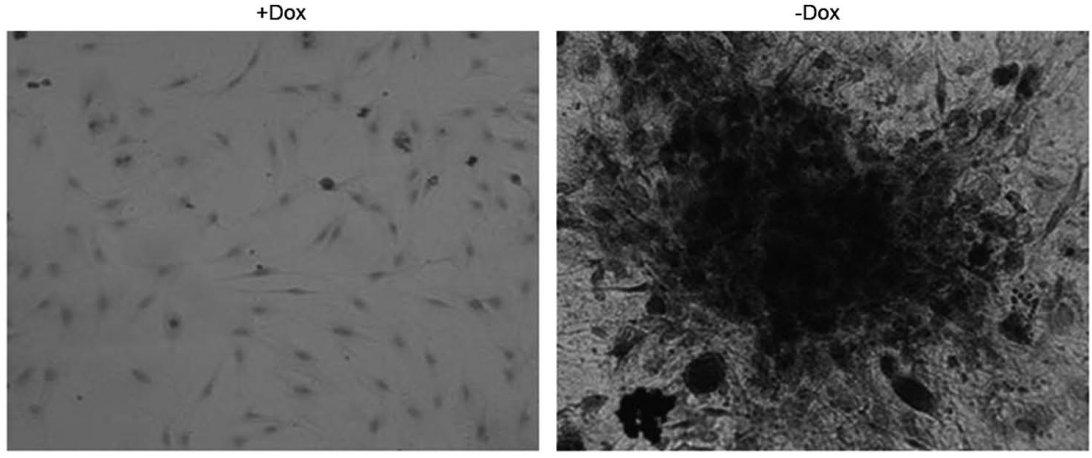

B

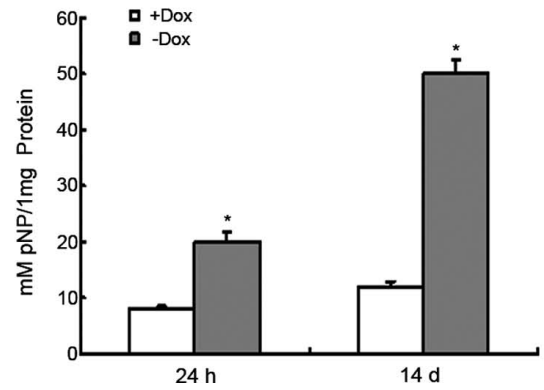

C

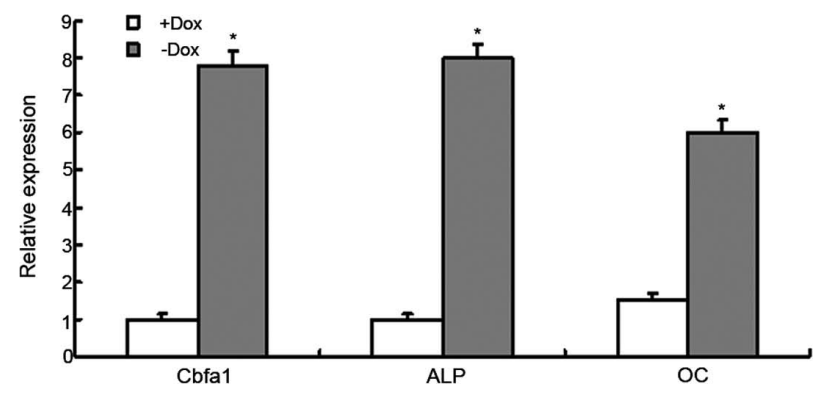

Figure 2. The role of Dox in controlling bFGF expression in differentiating MSCs. rAAV2-tet-off-bFGF-transduced MSCs were cultured with or without $1 \mu \mathrm{g} / \mathrm{ml}$ Dox. (A) ALP positive staining in the transduced cells in the absence of Dox (-Dox) compared with no staining at all in the presence of Dox (+Dox) 14 days after Dox treatment. (B) ALP activity in the cell lysate with or without Dox after $24 \mathrm{~h}$ or 14 days of Dox treatment. (C) The mRNA expression of osteogenic factors under induced conditions (-Dox) compared with the expression level in the non-induced state (+Dox) were detected by real-time RT-PCR 7 days after Dox administration. ${ }^{~} \mathrm{P}<0.01$ indicates significant difference compared with the cultures treated with Dox (+Dox).

13.0 was used to perform the statistical analysis. Multi-group one-way ANOVA, LSD pairwise comparison, and linear correlation and regression analysis were performed. $\mathrm{P}<0.05$ was considered a significant difference, and $\mathrm{P}<0.01$ was considered a markedly significant difference.

\section{Results}

Regulated bFGF expression and cell differentiation in vitro. Endogenous bFGF was weakly expressed in MSCs detected by real-time RT-PCR (Fig. 1A). The bFGF mRNA level was significantly increased in the rAAV2-transduced tet-off-bFGF group as compared to the non-transduction group (Fig. 1A). bFGF expression decreased as Dox dosage increased. bFGF expression decreased to a minimum as the Dox concentration reached $1 \mu \mathrm{g} / \mathrm{ml}$ (Fig. 1A). The trend of decreased bFGF expression was also confirmed by Western blotting (Fig. 1B and C). Therefore, $1 \mu \mathrm{g} / \mathrm{ml}$ Dox was selected as the concentration that is needed to control expression of the transgene.

ALP positive staining was shown in the Dox treatment group and non-Dox treatment group after $24 \mathrm{~h}$ (data not shown) and 14 days of culture. In the Dox treatment group, no distinct ALP staining was observed, while significant mineralization nodules were observed in the non-Dox treatment group after 14 days of culture (Fig. 2A). ALP activity significantly increased in the non-Dox treatment group after $24 \mathrm{~h}$ and 14 days of culture compared with the Dox treatment group (Fig. 2B). In the non-Dox treatment group, the mRNA expression levels of the osteogenic factors Cbfa1, ALP, and OC were significantly higher than those in the Dox treatment group (Fig. 2C).
The adhesion and proliferation of MSCs on $\beta$-TCP. SEM analysis revealed that cells began to move to the surface of $\beta$-TCP $15 \mathrm{~h}$ after transplantation and adhered completely $72 \mathrm{~h}$ later; they stretched into polygonal shapes with branches (Fig. 3). The proliferation activity of $\beta$-TCP composite MSCs was significantly increased 7 days after the rAAV2-tet-offbFGF was transduced, with no difference between the (+Dox) and (-Dox) groups; however, the proliferation activity of no- $\beta$-TCP composite MSCs was significantly different when rAAV2-tet-off-bFGF was not transduced (Fig. 4). MSCs proliferated faster in the composite adherent group than in the adherent group, and the difference was not statistically significant (Fig. 4). Real-time RT-PCR showed that after three-dimensional culture, osteogenic factors were slightly increased in genetically modified cells but not significantly different as compared with other groups (data not shown).

Regulation of bFGF expression in genetically engineered MSCs modulates bone regeneration of skull defects. After 8 weeks, large areas of new bone formation were observed in the -Dox group D, while only a small amount of new bone formation was observed in the +Dox group D (Fig. 5A). The bone volume fraction was higher in the absence of Dox than in the presence of Dox in group D both at 2 and 8 weeks after surgery (Fig. 5B).

Representative images of bone regeneration and angiogenesis showed notable differences that characterized the tissue response to each implant (Fig. 6A). In the -Dox group D, new bone was formed 2 weeks later and the bone lacunae were large (Fig. 6Aa). A large number of new vessels surrounded the new bone and materials (Fig. 6Aa). Erosion of macrophages was 

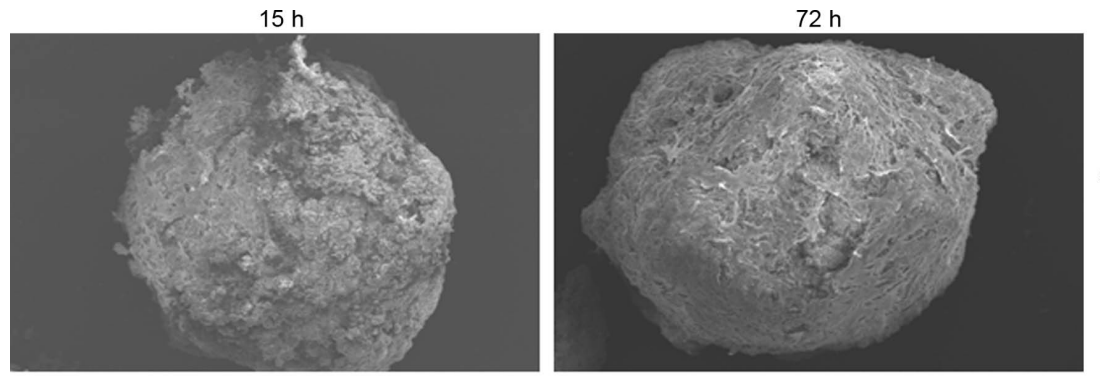

$500 \mu \mathrm{m}$
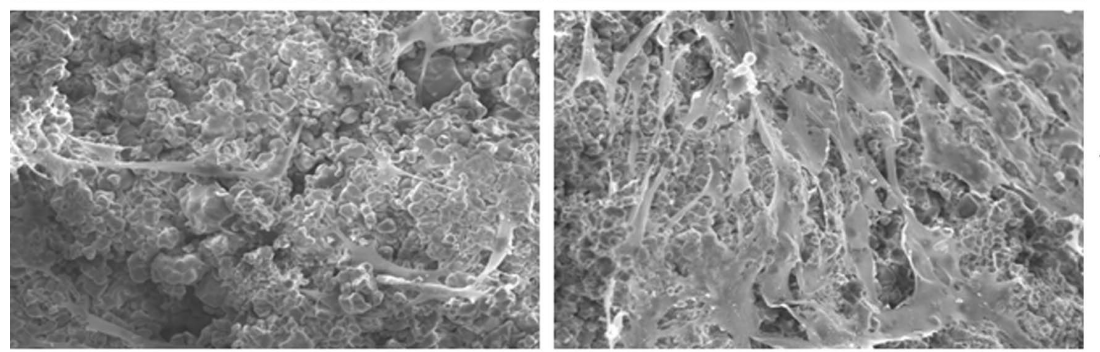

$100 \mu \mathrm{m}$
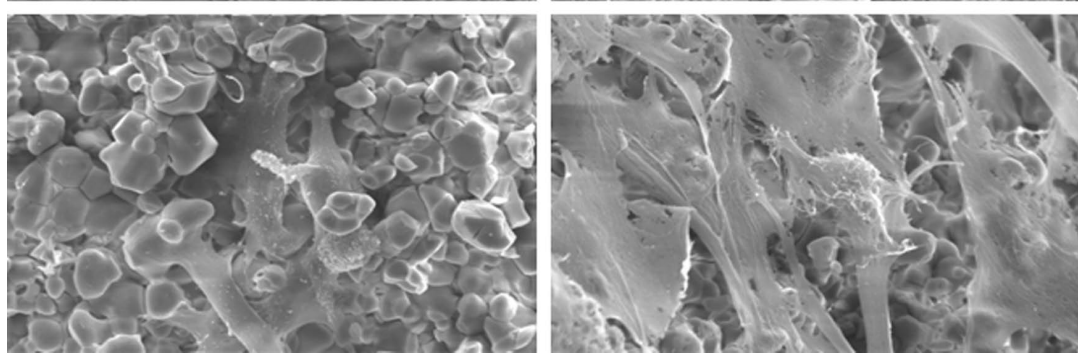

$30 \mu \mathrm{m}$

Figure 3. Scanning electron microscopy (SEM) images of adhesion and morphology of transduced MSCs on $\beta$-TCP granules after 15 or $72 \mathrm{~h}$ of culture.

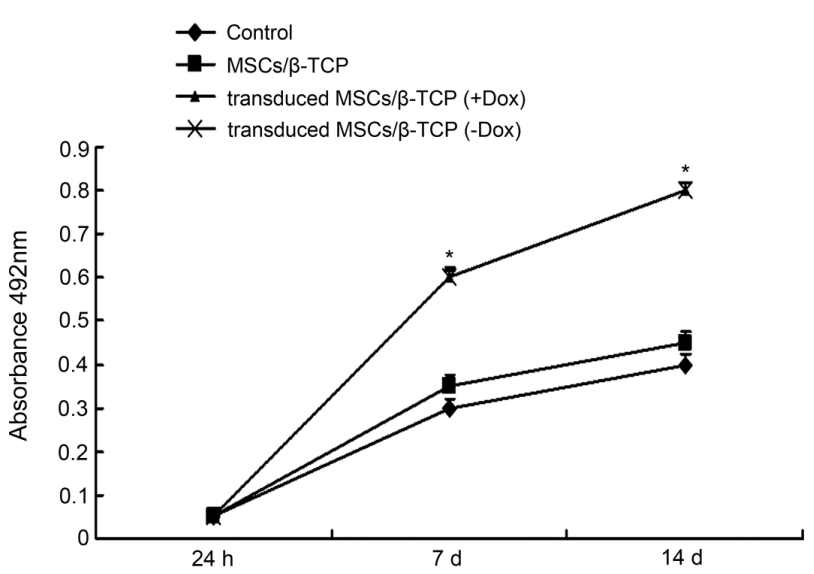

Figure 4. MTS detected the proliferation activities of three-dimensional cultured cells 24 h, 7 days, and 14 days after composition. ${ }^{*} \mathrm{P}<0.01$ indicates significant difference compared with the other two groups.

observed around the materials (Fig. 6Aa). Collagen fibers were ordered and more fibers existed in the active bone formation area (Fig. 6Ab). The fibroblasts changed into cubic osteoblasts and located on the surface of the new bone (Fig. 6Ab). Blood vessels were abundant (Fig. 6Ab). However, almost no new bone formation and angiogenesis were observed in the +Dox group D (Fig. 6Ac and d). In groups A, B, and C, no new bone formation was observed and the inflammation was significant (data not shown). Collagen fibers were sparse (data not shown). After 8 weeks of implantation, large areas of new bone fused
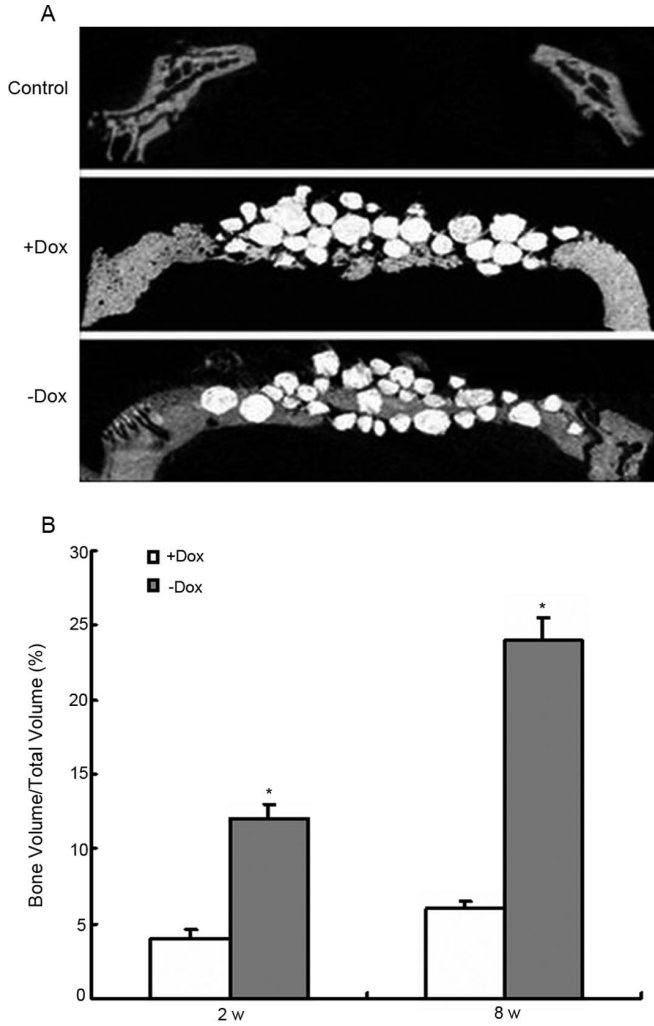

Figure 5. (A) MicroCT image reconstruction of calvarial defects in the control, +Dox and -Dox group D 8 weeks after surgery. (B) Quantitative analysis of the bone volume 2 and 8 weeks after surgery in the +Dox and the -Dox group D. ${ }^{*} \mathrm{P}<0.01$ indicates significant difference compared with the + Dox group D. 
A
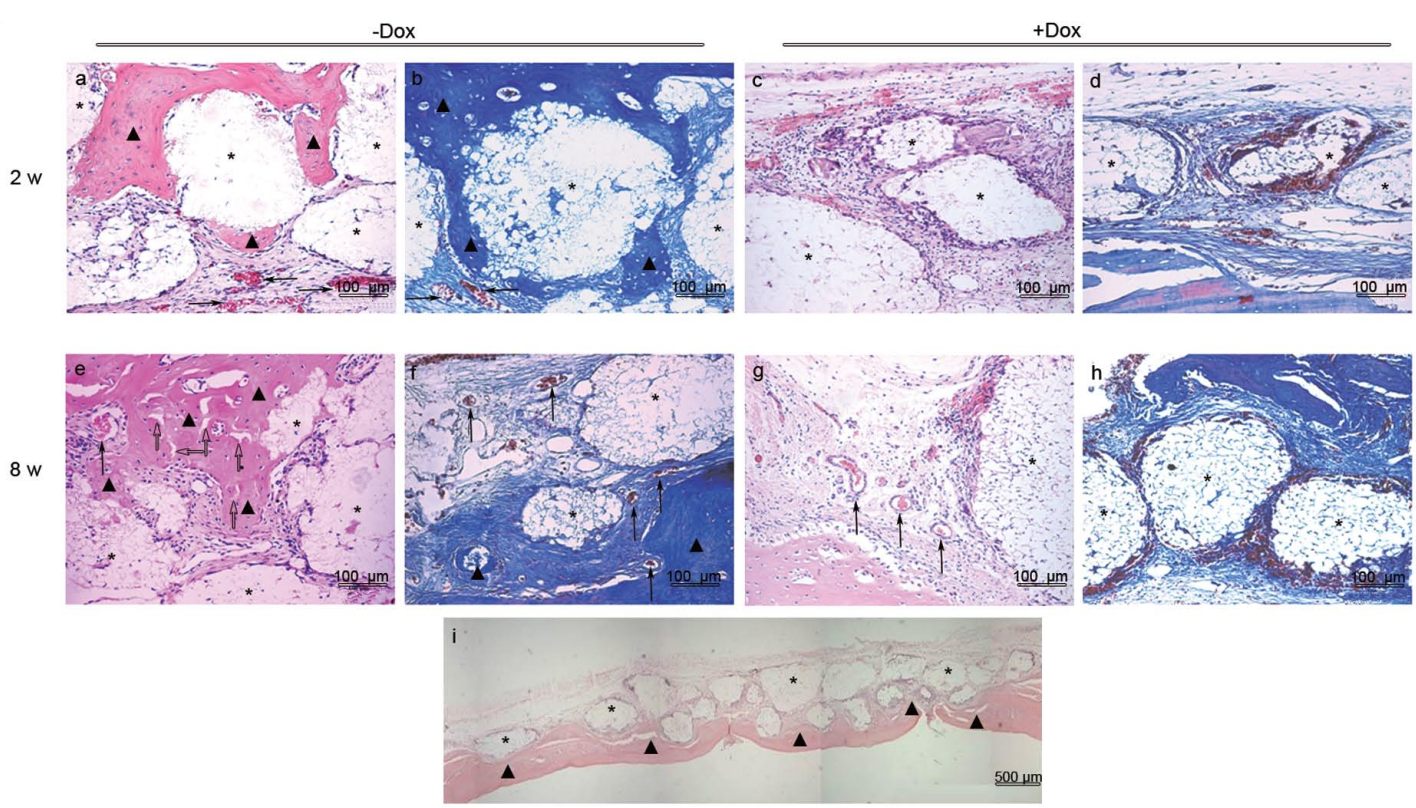

B

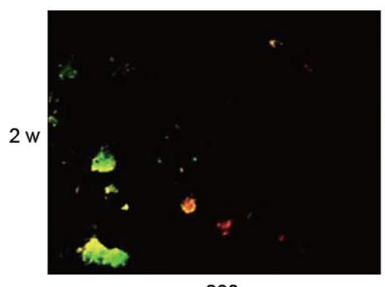

$\times 200$

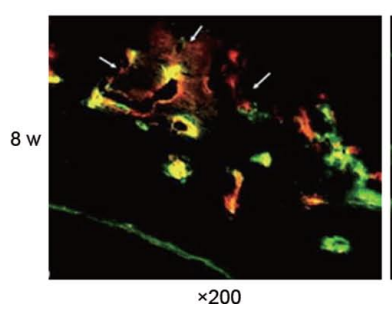

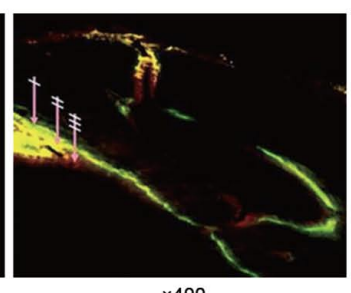

$\times 400$

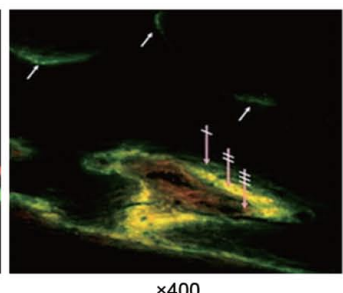

$\times 400$
C
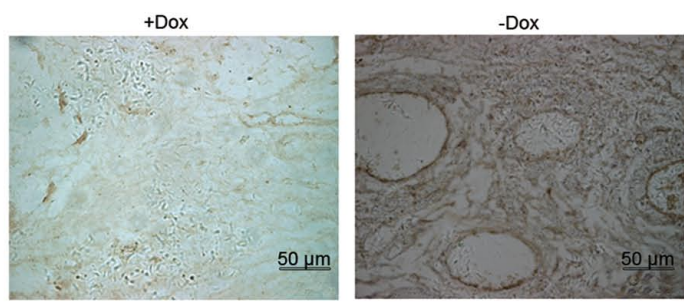

Figure 6. (A) New bone and blood vessel formation were analyzed by H\&E and Masson's staining 2 and 8 weeks after surgery. In the -Dox group D, a large number of new bone and blood vessels were visible 2 weeks after surgery (a, H\&E; b, Masson's); in the + Dox group D, no new bone was observed and new blood vessels were rarely observed (c, H\&E; d, Masson's). Eight weeks after surgery, the (-Dox) group had a significantly higher amount of new bone and blood vessels than the +Dox group (e and g, H\&E; f and h, Masson's). Multiple bone remodeling was observed (e and f). Panorama of the defect region in the -Dox group D 8 weeks after surgery (i) (original magnification $\mathrm{x} 40$ ). Triangles (A) indicate the new bone formation in direct contact with the bone substitutes Arrows show bone remodeling $(\uparrow)$ and blood vessels $(\uparrow)$ around the defects. Asterisks $(*)$ represent the materials in the zones of the calvarial defects. (original magnification x200). (B) Bone deposition was detected by fluorescent microscopy in the -Dox group D at 2 and 8 weeks. The arrows indicate the time sequence of bone deposition from left to right 2 and 8 weeks after surgery. (C) Immunohistochemical staining of factor VIII in tissue sections in the -Dox group D and the +Dox group D at 8 weeks after implantation (original magnification $\mathrm{x} 400$ ).

and bone remodeling was observed in the -Dox group D (Fig. 6Ae and $\mathrm{f}$ ). The materials were partially absorbed (Fig. 6Ae and $\mathrm{f}$ ). The vascular lumen was large, and the number of vessels continued increasing (Fig. 6Ae and f). The lacunae of the new bone gradually became small, and the blood vessels gradually extended into the lacunae to form the marrow cavity (Fig. 6A e and f). In the +Dox group D, only a small amount of newly formed bone was scattered near the margin of the old bone and materials and the neovascularization was also less than that in the -Dox group D (Fig. 6Ag and h). The panorama of the defect region in the -Dox group D 8 weeks after surgery was shown in Fig. 6Ai. In groups A, B, and C, no significant new bone formation was observed although inflammatory reactions were abundant (data not shown). In groups $\mathrm{A}, \mathrm{B}$, and C, Dox treatment and withdraw had no effect on the bone regeneration at 2 or 8 weeks (data not shown).

The different times and regions of bone mineral deposition were detected using dynamic fluorescence in the -Dox group D (Fig. 6B). The bone deposition time can be reflected sequentially as green, yellow, and red fluorescence. In 2 weeks, a small area of bone formation was observed only 4 days after the surgery in the absence of Dox. After 12 days, osteogenic activities increased and expanded. Apparent bone deposition occurred around the materials. After 8 weeks, the deposition of new bone increased and large areas of new bone deposition surrounded the surface of the materials. Immunohistochemical 
A

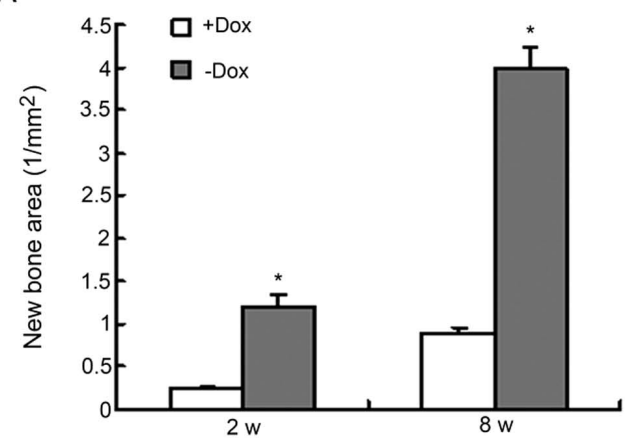

B

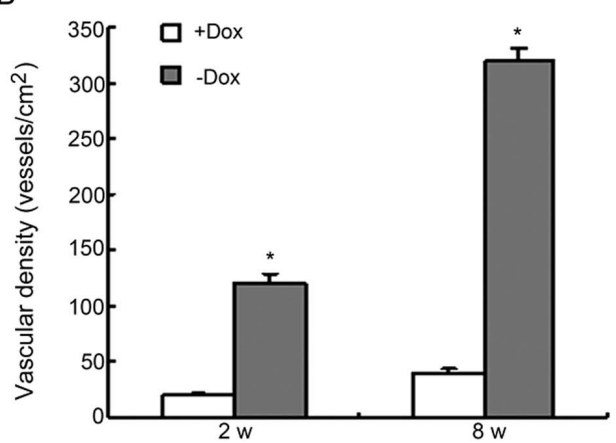

C

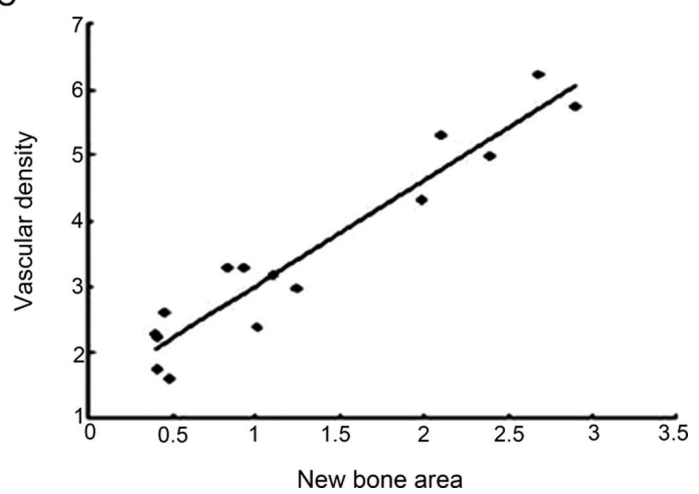

Figure 7. New bone area (A) and vascular density (B) were measured at 2 and at 8 weeks after surgery and was higher in the -Dox than in the + Dox group $D$. The correlation plot shows a positive correlation between the new bone area and the vascular density in the -Dox group D 2 to 8 weeks after surgery (C). ${ }^{*} \mathrm{P}<0.01$ indicates significant difference compared with the +Dox group $\mathrm{D}$.

staining for factor VIII in group D, revealed that blood vessel formation was inhibited with Dox treatment at 2 (data not shown) and 8 weeks after implantation (Fig. 6C).

The new bone area was significantly higher without Dox treatment than with Dox treatment (Fig. 7A). The vascular density had the same trend (Fig. 7B). New bone area and vascular density were positively correlated. A total of $91.5 \%$ of changes in the new bone area were related to changes in the vascular density in the -Dox group D at 2-8 weeks (Fig. 7C).

\section{Discussion}

During bone regeneration, osteogenesis and angiogenesis are two basic processes that may have important therapeutic potential (10). bFGF is an important factor for inducing osteogenic differentiation and angiogenesis (7-9). In the present study, we have shown that rAAV2-mediated bFGF could promote differentiation of MSCs in vitro and angiogenesis and osteogenesis in vivo. However, the process of tissue repair is complex, and the activation and expression of each gene have some temporal and dosage regulation. Non-controlled exogenous gene expression can cause excessive or abnormal tissue regeneration (18). Controlled exogenous gene expression in vivo can better guide tissue repair. In this study, rAAV2mediated bFGF expression was controlled by Dox in order to regulate bone regeneration.

Regulation of gene expression in gene therapy is critical. It can control the effects of gene therapy to some extent, and it can also simulate the physiological processes of tissue regeneration to achieve the best results (13). Effective regulation of bFGF is the key in our study. Our results showed that in the absence of Dox, rAAV2-tet-off-bFGF expressed a high amount of bFGF in MSCs. In addition, the expression levels of osteogenic factors Cbfa1, OC, and ALP also increased, suggesting that the osteoblast precursor cells were differentiating into osteoblasts. This was associated with the activation of downstream signaling in the pathways of bone morphogenetic proteins that are regulated by dramatically increased expression of bFGF $(19,20)$. In the presence of Dox, bFGF expression was shut off. When the final concentration of Dox reached $1 \mu \mathrm{g} / \mathrm{ml}$, only endogenous bFGF was expressed. In addition, the expression of Cbfa1, ALP, and OC was also inhibited. Therefore, this dosage was sufficient to minimize the expression of foreign genes, consistent with the in vitro results of other researchers $(11,21)$.

Dox could control bFGF expression in the rAAV2-tet-offbFGF system, which plays important roles in bone induction in vitro. However, monitoring tissue formation via regulated transgene expression is not a trivial issue. In our study, we used the rat critical-sized calvarial defect model (22) to explore the mechanisms of gene-controlled bone repair. Micro-CT showed that within 8 weeks of addition of Dox, bFGF expression was inhibited, and only minimal new bone formation was observed in the areas of skull defects, while in the bFGF treatment group, there was new bone formation 2 weeks after surgery and the materials and new bone were fully integrated 8 weeks after surgery. This not only confirmed the value of this model but also showed that bFGF can promote bone formation effectively. bFGF can bind to endothelial cell surface receptors and activate vascular immunogenicity (8). It can also activate the PI3K/Akt signaling pathway to inhibit the apoptosis of endothelial cells (23). It can induce chemotaxis of endometrial cells in order to induce the expression of a variety of proteolytic enzymes and collagenase, which in turn induce angiogenesis $(8,24)$. In group D, extensive angiogenesis was observed in the absence of Dox, 2 weeks after surgery. In addition, the vascular density was much greater in the non-Dox group than in the Dox treatment group 2 and 8 weeks after surgery. The increase was much more than that in the past models (25), suggesting the role of bFGF in pro-angiogenesis. In the presence of Dox, bFGF expression was inhibited. Thus, the angiogenesis pathway was blocked and blood vessel formation was significantly reduced. There was no significant difference between the +Dox group D and groups $\mathrm{A}, \mathrm{B}$, and $\mathrm{C}$.

Neovascularization in the early stages of bone defect repair is not the only channel for oxygen, nutrients, and 
metabolic products, but it also is an important way to deliver growth factors $(10,26)$. In our study, new bone formation was significantly higher in the -Dox group than in the + Dox treatment group among rAAV2-tet-off-bFGF-transduced MSCs, which could not be separated from the adequate blood supply due to expressed bFGF. The addition of Dox not only led to a time delay in the formation of blood vessels and new bone, but the numbers were also relatively small, indicating that Dox controlled not only angiogenesis but also bone regeneration by regulating bFGF expression. In group $\mathrm{D}$, $91.5 \%$ changes in the new bone area were related to vascular density during the 2 and 8 weeks after surgery when Dox was absent, indicating that bFGF activates vessels required for bone formation. Furthermore, bFGF can also induce the migration of mesenchymal and osteogenic cells (7). bFGF can act directly or in collaboration with relevant growth factors to stimulate cell proliferation and differentiation, and consequently bone regeneration $(7,20)$. Histology results showed that bone formation was significantly increased with strong bFGF expression. The increased bFGF per se promoted bone regeneration. In addition, the increased bFGF could promote angiogenesis, which also enhanced bone regeneration. These two aspects functioned together to promote bone regeneration. Unfortunately, 8 weeks after surgery, new bone did not fill the defects area. Increase in treatment time would achieve better results. Since the double labeling of Tet with alizarin red or calcein was reported, fluorescent labeling has been widely used to study bone dynamics $(27,28)$. Fluorescent dye injected into the body leads to calcium chelation and deposition, which can be observed with various fluorescence dyes at different excitation wavelengths. Thus, new bone deposition was dynamically observed in vivo. In group $\mathrm{D}$, dynamic bone remodeling occurred in the area surrounding the autogenous bone defects 2 weeks after surgery without the addition of Dox. Mineralization occurred from cancellous bone to cortical bone. Bone mineral deposition significantly increased 8 weeks after surgery. A large fluorescent area around the material was observed, but had an ill-defined limit of the different fluorescent colors, which was less continuous and clear than that of the long bone (29).

On the basis of the physiological mechanisms of craniofacial defects, bFGF was introduced for angiogenesis and bone regeneration in the early repair stage. In addition to the blank control, another two sets of the controls were also chosen to compare the intervention effects of bFGF on bone repair. In the control groups, no difference was observed, regardless of Dox addition, suggesting Dox had no effect on normal tissue. In the $\beta$-TCP and (MSCs $+\beta$-TCP) group, the formation of new blood vessels and bone was similar, and the new bone was increased compared with the control, although not significantly. MSCs themselves are involved in promoting vascular and bone formation (30), but in our study, this phenomenon was not observed. Because of the lack of osteogenic factors, bone formation was less than that in the bFGF/MSCs $+\beta-\mathrm{TCP}$ group, and inflammation was significant. The scaffold materials provided adequate space and support for the new bone and met the basic requirements of tissue engineering materials. $\beta$-TCP has good biocompatibility, bone conduction, better degradation, and pro-metabolism and growth $(31,32)$. Therefore, it has been widely used in clinical and basic research. In our study, $\beta$-TCP composite transgenic cells were implanted in vivo to evaluate the introduction of controllable gene-enhanced bone tissue engineering in order to promote bone regeneration. Cell adhesion to the scaffolds is the key step for cell migration and differentiation. In our study, $\beta$-TCP was the composite material for transgenic MSCs, and MSCs proliferated and adhered well to the material, demonstrating that the porous structure provided sufficient space and nutrient supply. Furthermore, rAAV2-tet-off-bFGF did not produce any damage to MSCs and bFGF can promote the proliferation of MSCs on the $\beta$-TCP. Real-time RT-PCR analysis revealed that the osteogenic factors in the three-dimensional culture were slightly increased as compared to in the adherent cells, confirming that $\beta$-TCP had bone conduction but lacked bone induction ability. When only $\beta$-TCP was implanted, it produced slightly more bone than the control, but the difference was not significant. This also proved that $\beta$-TCP lacks bone induction ability. However, the amount of new bone was significantly lower in the $\beta$-TCP group than in the $\mathrm{bFGF} / \mathrm{MSCs}+\beta$-TCP group, underlining the role of bFGF in promoting bone regeneration.

In summary, our results demonstrate that rAAV2-mediated bFGF transfer can promote angiogenesis and bone formation well enough for the repair of rat skull defects. In addition, through Tet-off regulation of bFGF expression, angiogenesis and bone formation can effectively be controlled in vivo. This strengthened the notion that through regulating the expression of target genes that are required to simulate the regeneration process, better bone regeneration can be achieved. We plan to continue performing time- and dose-dependent experiments to better understand how gene expression can be effectively controlled. Our experiments will provide the basis for multistage and multi-gene combination therapy.

\section{Acknowledgements}

This study was supported by the National Natural Sciences Foundation of China (No. 30572065/C03031103).

\section{References}

1. Kneser U, Schaefer DJ, Polykandriotis E and Horch RE: Tissue engineering of bone: the reconstructive surgeon's point of view. J Cell Mol Med 10: 7-19, 2006.

2. Goiato MC, Anchieta RB, Pita MS and dos Santos DM: Reconstruction of skull defects: currently available materials. J Craniofac Surg 20: 1512-1518, 2009.

3. Drosse I, Volkmer E, Capanna R, De Biase P, Mutschler W and Schieker M: Tissue engineering for bone defect healing: an update on a multi-component approach. Injury 39 (Suppl. 2): S9-S20, 2008.

4. Betz VM, Betz OB, Harris MB, Vrahas MS and Evans CH: Bone tissue engineering and repair by gene therapy. Front Biosci 13: 833-841, 2008

5. Hao J, Varshney RR and Wang DA: Engineering osteogenesis and chondrogenesis with gene-enhanced therapeutic cells. Curr Opin Mol Ther 11: 404-410, 2009.

6. Zellin G: Growth factors and bone regeneration. Implications of barrier membranes. Swed Dent J (Suppl) 129: 7-65, 1998.

7. Radomsky ML, Thompson AY, Spiro RC and Poser JW: Potential role of fibroblast growth factor in enhancement of fracture healing. Clin Orthop Relat Res 355 (Suppl.): S283-293, 1998.

8. Nomi M, Miyake H, Sugita Y, Fujisawa M and Soker S: Role of growth factors and endothelial cells in therapeutic angiogenesis and tissue engineering. Curr Stem Cell Res Ther 1: 333-343, 2006. 
9. Kim MS, Bhang SH, Yang HS, et al: Development of functional fibrous matrices for the controlled release of basic fibroblast growth factor to improve therapeutic angiogenesis. Tissue Eng (Part A) 16: 2999-3010, 2010

10. Kanczler JM and Oreffo RO: Osteogenesis and angiogenesis: the potential for engineering bone. Eur Cell Mater 15: 100-114, 2008

11. Gafni Y, Pelled G, Zilberman Y, et al: Gene therapy platform for bone regeneration using an exogenously regulated, $\mathrm{AAV}-2$-based gene expression system. Mol Ther 9: 587-595, 2004.

12. Li Q, Dai J and Rabie AB: Recombinant adeno-associated virus serotype 2 (rAAV2)-An efficient vector for gene delivery in condylar cartilage, glenoid fossa and TMJ disc in an experimental study in vivo. Arch Oral Biol 54: 943-950, 2009.

13. Gossen M and Bujard H: Tight control of gene expression in mammalian cells by tetracycline-responsive promoters. Proc Natl Acad Sci USA 89: 5547-5551, 1992.

14. Han Y, Chang QA, Virag T, West NC, George D, Castro MG and Bohn MC: Lack of humoral immune response to the tetracycline (Tet) activator in rats injected intracranially with Tet-off rAAV vectors. Gene Ther 17: 616-625, 2010.

15. Bianco P, Riminucci M, Gronthos S and Robey PG: Bone marrow stromal stem cells: nature, biology, and potential applications. Stem Cells 19: 180-192, 2001.

16. Hutmacher DW and Cool S: Concepts of scaffold-based tissue engineering - the rationale to use solid free-form fabrication techniques. J Cell Mol Med 11: 654-669, 2007.

17. Ponnazhagan S, Yoder MC and Srivastava A: Adeno-associated virus type 2-mediated transduction of murine hematopoietic cells with long-term repopulating ability and sustained expression of a human globin gene in vivo. J Virol 71: 3098-3104, 1997.

18. Franceschi RT: Biological approaches to bone regeneration by gene therapy. J Dent Res 84: 1093-1103, 2005.

19. Pri-Chen S, Pitaru S, Lokiec F and Savion N: Basic fibroblast growth factor enhances the growth and expression of the osteogenic phenotype of dexamethasone-treated human bone marrow-derived bone-like cells in culture. Bone 23: 111-117, 1998.

20. Naganawa T, Xiao L, Coffin JD, Doetschman T, Sabbieti MG Agas D and Hurley MM: Reduced expression and function of bone morphogenetic protein-2 in bones of Fgf2 null mice. J Cell Biochem 103: 1975-1988, 2008.
21. Moutsatsos IK, Turgeman G, Zhou S, et al: Exogenously regulated stem cell-mediated gene therapy for bone regeneration. Mol Ther 3: 449-461, 2001.

22. Gosain AK, Song L, Yu P, Mehrara BJ, Maeda CY, Gold LI and Longaker MT: Osteogenesis in cranial defects: reassessment of the concept of critical size and the expression of TGF-beta isoforms. Plast Reconstr Surg 106: 360-372, 2000.

23. Yu P, Passam FH, Yu DM, Denyer G and Krilis SA: Beta2glycoprotein I inhibits vascular endothelial growth factor and basic fibroblast growth factor induced angiogenesis through its amino terminal domain. J Thromb Haemost 6: 1215-1223, 2008.

24. Cohen MM Jr: The new bone biology: pathologic, molecular, and clinical correlates. Am J Med Genet A 140: 2646-2706, 2006

25. Leach JK, Kaigler D, Wang Z, Krebsbach PH and Mooney DJ: Coating of VEGF-releasing scaffolds with bioactive glass for angiogenesis and bone regeneration. Biomaterials 27: 3249-3255, 2006.

26. Ishida $\mathrm{K}$, Matsumoto $\mathrm{T}$, Sasaki $\mathrm{K}$, et al: Bone regeneration properties of granulocyte colony-stimulating factor via neovascularization and osteogenesis. Tissue Eng Part A 16: 3271-3284, 2010.

27. Harris WH: A microscopic method of determining rates of bone growth. Nature 188: 1038-1039, 1960.

28. Suzuki HK, Mathews A: Two-color fluorescence labeling of mineralizing tissues with tetracycline and 2,4-bis [N,N'-di(carbomethyl)aminomethyl] fluorescein. Stain Technol 41: 57-60, 1966.

29. Lee TC, Arthur TL, Gibson LJ and Hayes WC: Sequential labelling of microdamage in bone using chelating agents. J Orthop Res 18: 322-325, 2000.

30. Wu Y, Chen L, Scott PG and Tredget EE: Mesenchymal stem cells enhance wound healing through differentiation and angiogenesis. Stem Cells 25: 2648-2659, 2007.

31. Zerbo IR, Bronckers AL, de Lange $\mathrm{G}$ and Burger EH: Localisation of osteogenic and osteoclastic cells in porous betatricalcium phosphate particles used for human maxillary sinus floor elevation. Biomaterials 26: 1445-1451, 2005.

32. Artzi Z, Weinreb M, Givol N, Rohrer MD, Nemcovsky CE, Prasad HS and Tal H: Biomaterial resorption rate and healing site morphology of inorganic bovine bone and beta-tricalcium phosphate in the canine: a 24-month longitudinal histologic study and morphometric analysis. Int J Oral Maxillofac Implants 19: 357-368, 2004. 